\title{
On Kadison's Similarity Problem for Homomorphisms of the Algebra of Complex Polynomials
}

\author{
Joachim Moussounda Mouanda \\ Blessington Christian University, Nkayi, Republic of Congo \\ Email:mmoussounda@yahoo.fr
}

How to cite this paper: Mouanda, J.M. (2021) On Kadison's Similarity Problem for Homomorphisms of the Algebra of Complex Polynomials. Advances in Pure Mathematics, 11, 755-770.

https://doi.org/10.4236/apm.2021.119050

Received: June 7, 2021

Accepted: September 19, 2021

Published: September 22, 2021

Copyright $\odot 2021$ by author(s) and Scientific Research Publishing Inc. This work is licensed under the Creative Commons Attribution International License (CC BY 4.0). http://creativecommons.org/licenses/by/4.0/

\begin{abstract}
We prove that every homomorphism of the algebra $\mathcal{P}_{n}$ into the algebra of operators on a Hilbert space is completely bounded. We show that the contractive homomorphism introduced by Parrott, which is not completely contractive, is completely bounded (similar to a completely contractive homomorphism). We also show that homomorphisms of the algebra $\mathcal{P}_{n}$ generate completely positive maps over the algebras $C\left(\mathbb{T}^{n}\right)$ and $M_{2}\left(C\left(\mathbb{T}^{n}\right)\right)$.
\end{abstract}

\section{Keywords}

Inequalities for Sums, Fourier Coefficients, Operator Theory, Polynomials

\section{Introduction}

In 1950, Dixmier established the following similarity problem: Whether or not bounded representations of groups are necessarily similar to unitary representations. The same year, Dixmier proved that every uniformly bounded representation of an amenable group $\mathrm{G}$ on a Hilbert space $\mathrm{H}$ is similar to a unitary representation of G on H [1]. However, in 1955, L. Ehrenpreis and F. Mautner [2] gave examples showing that this result fails when the amenability assumption is removed. Therefore, there exist bounded homomorphisms of groups into the group of invertible operators on a Hilbert space, which are not similar to unitary representations of the group. In 1955, motivated by Dixmier's similarity problem, the analogous problem for representations of $C$-algebras was introduced by Kadison. Kadison's similarity problem asks whether every bounded unital representation of a unital $C$-algebra $\mathcal{A}$ on a Hilbert space $\mathrm{H}$ is similar to a ${ }^{*}$-representation of $\mathcal{A}$ on $\mathrm{H}$ [3]. In 1960, Kunze-Stein proved that Dix- 
mier's question has a negative answer, that is, there exist bounded representations of groups $\left(S L_{2}(\mathbb{R})\right)$ that are not similar to unitary representations [4]. In the early 1980's, motivated by the work of Wittstock and Haagerup, the theory of completely positive maps was extended to the family of completely bounded maps by many researchers [5]. The theory of completely bounded maps has many deep connections to similarity questions. In 1981, Christensen solves the original problem for irreducible representations (so, each vector is cyclic for the image) [6]. In 1983, Haagerup gave a positive answer to Kadison's similarity problem for cyclic representations and proved that a bounded representation $\pi$ of a $C$-algebra $\mathcal{A}$ on $\mathrm{H}$ is similar to a ${ }^{*}$-representation if and only if $\pi$ is completely bounded. The result of Haagerup shows that Kadison's similarity problem is equivalent to determining whether or not bounded homomorphisms are necessarily completely bounded. There are several important cases where bounded homomorphisms of a $C$-algebra are completely bounded. Haagerup proves a number of other results. For example, he settles the problem completely for $C$-algebras that admit no trace. He observes that the problem reduces to studying representations of von Neumann algebras. Thus the problem is settled positively for von Neumann algebras with no central summand of finite type [7]. In 1984, Vern Paulsen generalized Haagerup's result to the non-self-adjoint case. He proved that every unital homomorphism of an operator algebra is a completely bounded homomorphism if and only if it is similar to a completely contractive homomorphism [8]. However, contractive homomorphisms need not be completely contractive. Parrott introduced in [9] an example of a contractive homomorphism which is not a completely contractive homomorphism. In 1986, Christensen settles the problem for factors of type $I I_{1}$ with property $\Gamma$ [10]. We say that the $C$-algebra $\mathcal{A}$ has the similarity property if the similarity problem has a positive answer for $\mathcal{A}$. In 1996, Kirchberg showed that Kadison's similarity problem is equivalent to another important open question, the derivation problem, itself a crucial problem in the cohomology theory of operator algebras [11]. In 1999, motivated by the similarity problem, Pisier introduced the notion of the length $\ell(\mathcal{B})$ of an operator algebra $\mathcal{B}$ in [12] and examined its properties in [13] [14]. This integer arises from the ability to write matrices over $\mathcal{B}$ as products of bounded length, where the constituent factors alternate between scalar matrices and diagonal matrices over $\mathcal{B}$. If such decompositions do not exist then $\ell(\mathcal{B})=\infty$. An easy consequence of finite length is that all bounded homomorphism of $\ell(\mathcal{B})$ into any $\mathrm{B}(\mathrm{H})$ are completely bounded, which solves the similarity problem for such algebras, and is indeed equivalent to it. In 2010, Christensen, Sinclair, Smith and White examine connections between the theory of perturbations and Kadison's similarity problem [15]. At the present time, the similarity problem is still open. The Factorization of matrices of complex polynomials of several variables, in terms of products of Matrices of polynomials of two variables with positive matrices as Fourier coefficients, gives us some light on von Neumann's inequality for matrices of complex polynomials 
of several variables.

Kadison's similarity problem has many engineering applications.

In this paper, we prove that homomorphisms of the algebra $\mathcal{P}_{n}$ are completely bounded. We show that Parrott's contractive homomorphism, which is not completely contractive, is completely bounded (similar to a completely contractive homomorphism). We also prove that every homomorphism of the algebra $\mathcal{P}_{n}$ generates completely positive maps over the algebras $C\left(\mathbb{T}^{n}\right)$ and $M_{2}\left(C\left(\mathbb{T}^{n}\right)\right)$.

\section{Main Result}

In this section, we prove our main result and we show that Parrott's contractive homomorphism is similar to a completely contractive homomorphism over the algebra of complex polynomials over $\mathbb{D}^{n}$. We construct completely positive maps over the algebras $C\left(\mathbb{T}^{n}\right)$ and $M_{2}\left(C\left(\mathbb{T}^{n}\right)\right)$. Given

$$
f\left(z_{1}, \cdots, z_{n}\right)=\sum_{\left(k_{1}, \cdots, k_{n}\right) \in S_{f}} \hat{f}\left(k_{1}, \cdots, k_{n}\right) z_{1}^{k_{1}} \cdots z_{n}^{k_{n}},
$$

a complex polynomial over $\mathbb{D}^{n}$. Then

$$
\|f\|_{\infty}=\sup \left\{\left|f\left(z_{1}, \cdots, z_{n}\right)\right|:\left|z_{1}\right|=\cdots=\left|z_{n}\right|=1\right\} .
$$

Let $\mathcal{P}_{n}$ denote the algebra of complex polynomials over $\mathbb{D}^{n}$. Given

$$
f_{i, j}\left(z_{1}, \cdots, z_{n}\right)=\sum_{\left(k_{1}, \cdots, k_{n}\right) \in S_{f_{i, j}}} \hat{f}_{i, j}\left(k_{1}, \cdots, k_{n}\right) z_{1}^{k_{1}} \cdots z_{n}^{k_{n}}, i, j=1, \cdots, k ;
$$

complex polynomials over $\mathbb{D}^{n}$. Then

$$
\left\|\left(f_{i, j}\right)_{i, j=1}^{k}\right\|_{\infty}=\sup \left\{\left\|\left(f_{i, j}\left(z_{1}, \cdots, z_{n}\right)\right)_{i, j=1}^{k}\right\|_{M_{k}}:\left|z_{i}\right| \leq 1\right\} .
$$

Denote by

$$
S_{F}=\bigcup_{i=1}^{k} \bigcup_{j=1}^{k} S_{f_{i, j}}
$$

Then the $k \times k$-matrix $F\left(z_{1}, \cdots, z_{n}\right)=\left(f_{i, j}\left(z_{1}, \cdots, z_{n}\right)\right)_{i, j=1}^{k}$ can be written as

$$
F\left(z_{1}, \cdots, z_{n}\right)=\sum_{\left(k_{1}, \cdots, k_{n}\right) \in S_{F}}\left(\hat{f}_{i, j}\left(k_{1}, \cdots, k_{n}\right)\right)_{i, j=1}^{k} z_{1}^{k_{1}} \cdots z_{n}^{k_{n}} .
$$

The $k \times k$-matrix $F\left(z_{1}, \cdots, z_{n}\right)$ can be regarded as a polynomial over $\mathbb{D}^{n}$ with matrix coefficients. In this case, the set

$$
\left\{\left(\hat{f}_{i, j}\left(k_{1}, \cdots, k_{n}\right)\right)_{i, j=1}^{k}:\left(k_{1}, \cdots, k_{n}\right) \in S_{F}\right\}
$$

is the set of Fourier coefficients of $F\left(z_{1}, \cdots, z_{n}\right)$. Let us set

$$
\|F\|_{u, k}=\sup \left\|\left(f_{i, j}\left(T_{1}, \cdots, T_{n}\right)\right)_{i, j=1}^{k}\right\|
$$

where the supremum is taken over the family of all $n$-tuples of commuting contractions on all Hilbert spaces. It is easy to see that $\|F\|_{u, k}$ is finite, since it is bounded by the sum of the norm operators of the Fourier coefficients of $f$, and that this quantity defines a norm on the algebra $M_{k}\left(\mathcal{P}_{n}\right)$ of matrices of polynomials 
over $\mathbb{D}^{n}$. For each polynomial $P$ in $M_{k}\left(\mathcal{P}_{n}\right)$, there is always an $n$-tuple of contractions where this supremum is achieved. Therefore, $\left(M_{k}\left(\mathcal{P}_{n}\right),\|\cdot\|_{u, k}\right)$ and $\left(M_{k}\left(\mathcal{P}_{n}\right),\|\cdot\|_{\infty}\right)$ are normed algebras. Let

$$
F\left(z_{1}, \cdots, z_{n}\right)=\sum_{\left(k_{1}, \cdots, k_{n}\right) \in S_{F}}\left(\hat{f}_{i, j}\left(k_{1}, \cdots, k_{n}\right)\right)_{i, j=1}^{k} z_{1}^{k_{1}} \cdots z_{n}^{k_{n}}
$$

be a matrix of complex polynomials over $\mathbb{D}^{n}$. Define a function

$$
\Pi_{k}: M_{k}\left(\mathcal{P}_{n}\right) \rightarrow \mathbb{R}^{+}
$$

by setting

$$
\Pi_{k}(F)=\sum_{\left(k_{1}, \cdots, k_{n}\right) \in S_{F}}\left\|\left(\hat{f}_{i, j}\left(k_{1}, \cdots, k_{n}\right)\right)_{i, j=1}^{k}\right\|_{M_{k}}, \forall F \in M_{k}\left(\mathcal{P}_{n}\right) .
$$

We can check that $\Pi_{k}$ defines a norm on $M_{k}\left(\mathcal{P}_{n}\right)$. Therefore, $\left(M_{k}\left(\mathcal{P}_{n}\right), \Pi_{k}\right)$ is also a normed algebra.

The Hahn-Banach extension property states that a vector subspace $\mathrm{Y}$ of $\mathrm{X}$ has the extension property if any continuous linear functional on $\mathrm{Y}$ can be extended to a continuous linear functional on X. The Hahn-Banach theorem is a useful tool in Functional Analysis. It allows the extension of bounded linear functional defined on a subspace of some vector space to the whole space. The proof of the Hahn-Banach type extension theorem for completely bounded maps introduced by Wittstock can be found in [5].

Theorem 2.1. (Wittstock's Extension Theorem). Let $\mathcal{A}$ be a $C^{*}$-algebra, $\mathcal{M}$ a subspace of $\mathcal{A}$, and let

$$
\phi: \mathcal{M} \rightarrow B(H)
$$

be completely bounded. Then there exists a completely bounded map

$$
\varphi: \mathcal{A} \rightarrow B(H),
$$

which extends $\phi$, with $\|\phi\|_{c b}=\|\varphi\|_{c b}$.

Kadison's similarity problem is that every bounded homomorphism of a $C^{*}$ algebra into $B(H)$ is similar to a ${ }^{*}$-homomorphism. Haagerup proved that every unital bounded homomorphism of a $C^{*}$-algebra is similar to a ${ }^{*}$-homomorphism if and only if it is a completely bounded homomorphism [7].

Theorem 2.2. [7]. Let $\mathcal{A}$ be a $C^{*}$-algebra with unit and let $\rho: \mathcal{A} \rightarrow B(H)$ be a bounded, unital homomorphism. Then $\rho$ is similar to $a^{*}$-homomorphism if and only if $\rho$ is completely bounded. Moreover, if $\rho$ is completely bounded, then there exists a similarity $S$ with $S^{-1} \rho()$.$S is a -homomorphism and$

$$
\left\|S^{-1}\right\| \cdot\|S\|=\|\rho\|_{c b} .
$$

Haagerup's result shows that Kadison's similarity problem is equivalent to determining whether or not bounded homomorphisms are necessarily completely bounded. Paulsen generalized Haaerup's result to the non-self-adjoint case and he proved several results on completely bounded maps over unital $C^{*}$-algebras [8].

Theorem 2.3. [5]. Let $\mathcal{A}$ be an operator algebra and let

$$
\rho: \mathcal{A} \rightarrow B(H)
$$


be unital completely bounded homomorphism. Then there exists an invertible operator $S$ with $\left\|S^{-1}\right\| \cdot\|S\|=\|\rho\|_{c b}$ such that $S^{-1} \rho()$.$S is a completely contrac-$ tive homomorphism. Moreover,

$$
\|\rho\|_{c b}=\inf \left\{\left\|R^{-1}\right\| \cdot\|R\|: R^{-1} \rho(.) R \text { is completely contractive }\right\} .
$$

Theorem 2.4. Let $\mathcal{A}$ be a $C^{*}$-algebra with unit and let $\phi: \mathcal{A} \rightarrow B(H)$ be completely bounded. Then there exist completely positive maps

$$
\theta_{i}: \mathcal{A} \rightarrow B(H),
$$

with $\left\|\theta_{i}\right\|_{c b}=\|\phi\|_{c b}, i=1,2$ such that the map $\psi: M_{2}(\mathcal{A}) \rightarrow B(H \oplus H)$ given by

$$
\psi\left[\left[\begin{array}{ll}
a & b \\
c & d
\end{array}\right]\right]=\left[\begin{array}{ll}
\theta_{1}(a) & \phi(b) \\
\phi^{*}(c) & \theta_{2}(d)
\end{array}\right]
$$

is completely positive. Moreover, if $\|\phi\|_{c b} \leq 1$, then we may take

$$
\theta_{1}(1)=\theta_{2}(1)=I_{H} \text {. }
$$

The structure of the Fourier coefficients of a complex polynomial $f\left(z_{1}, \cdots, z_{n}\right)$ over $\mathbb{D}^{n}$ is linked to the properties of $f\left(z_{1}, \cdots, z_{n}\right)$. For example, if

$$
f\left(z_{1}, \cdots, z_{n}\right)=\sum_{\left(k_{1}, \cdots, k_{n}\right) \in S_{F}}\left|a\left(k_{1}, \cdots, k_{n}\right)\right| z_{1}^{k_{1}} \cdots z_{n}^{k_{n}}
$$

is a complex polynomial with a positive real number as Fourier coefficients, then

$$
\|f\|_{\infty}=\sum_{\left(k_{1}, \cdots, k_{n}\right) \in S_{F}}\left|a\left(k_{1}, \cdots, k_{n}\right)\right| .
$$

In this case,

$$
\left\|f\left(T_{1}, \cdots, T_{n}\right)\right\| \leq \sum_{\left(k_{1}, \cdots, k_{n}\right) \in S_{F}}\left|a\left(k_{1}, \cdots, k_{n}\right)\right|=\|f\|_{\infty}
$$

for any $n$-tuple $\left\{T_{1}, \cdots, T_{n}\right\}$ of commuting contractions on a Hilbert space. Complex polynomials over $\mathbb{D}^{n}$ with positive real numbers as Fourier coefficients allow the construction of complex polynomials over $\mathbb{D}^{n}$.

Remark 2.5. Let

$$
f\left(z_{1}, \cdots, z_{n}\right)=\sum_{\left(k_{1}, \cdots, k_{n}\right) \in S_{F}} \hat{f}\left(k_{1}, \cdots, k_{n}\right) z_{1}^{k_{1}} \cdots z_{n}^{k_{n}}
$$

be a complex polynomial over $\mathbb{D}^{n}$. Then $\forall\left(k_{1}, \cdots, k_{n}\right) \in S_{F}$ there exists a constant $\alpha\left(k_{1}, \cdots, k_{n}\right) \in \mathbb{N}, \alpha\left(k_{1}, \cdots, k_{n}\right) \neq 0$, such that

$$
\left|2 \hat{f}\left(k_{1}, \cdots, k_{n}\right)+\alpha\left(k_{1}, \cdots, k_{n}\right)\right|^{2} \neq 0 .
$$

Let

$$
g_{j}\left(f\left(z_{1}, \cdots, z_{n}\right)\right)=\sum_{\left(k_{1}, \cdots, k_{n}\right) \in S_{F}}\left|2 \hat{f}\left(k_{1}, \cdots, k_{n}\right)+\alpha\left(k_{1}, \cdots, k_{n}\right)\right|^{2}\left[z_{j}^{k_{j}} z_{j+1}^{k_{j+1}}+z_{j}^{k_{j}}\right],
$$

$j=1, \cdots, n-1$, be complex polynomials over $\mathbb{D}^{n}$. Then there exists a complex polynomial $p^{f}\left(z_{1}, \cdots, z_{n}\right)$ over $\mathbb{D}^{n}$ such that

$$
\prod_{j=1}^{n-1} g_{j}\left(f\left(z_{1}, \cdots, z_{n}\right)\right)=2 f\left(z_{1}, \cdots, z_{n}\right)+p^{f}\left(z_{1}, \cdots, z_{n}\right) .
$$

It is possible to factorize matrices of complex polynomials. Let 


$$
Q\left(z_{1}, z_{2}, z_{3}\right)=\sum_{\left(k_{1}, k_{2}, k_{3}\right) \in S_{F}} \hat{Q}\left(k_{1}, k_{2}, k_{3}\right) z_{1}^{k_{1}} z_{2}^{k_{2}} z_{3}^{k_{3}}
$$

be a matrix of complex polynomials of three variables of $M_{k}\left(\mathcal{P}_{3}\right)$. Then $\forall\left(k_{1}, k_{2}, k_{3}\right) \in S_{F}$ there exists a constant $\alpha\left(k_{1}, k_{2}, k_{3}\right) \in \mathbb{N}, \alpha\left(k_{1}, k_{2}, k_{3}\right) \neq 0$, such that

$$
\left|2 \hat{Q}\left(k_{1}, k_{2}, k_{3}\right)+\alpha\left(k_{1}, k_{2}, k_{3}\right) I_{k}\right|^{2} \neq 0 \text {. }
$$

Let us notice that $\left|2 \hat{Q}\left(k_{1}, k_{2}, k_{3}\right)+\alpha\left(k_{1}, k_{2}, k_{3}\right) I_{k}\right|^{2}$ is a positive matrix of complex polynomials and $\alpha\left(k_{1}, k_{2}, k_{3}\right)$ is not unique. Denote by

$$
B_{1}\left(z_{1}, z_{2}, z_{3}\right)=\sum_{\left(k_{1}, k_{2}, k_{3}\right) \in S_{F}}\left|\hat{Q}\left(k_{1}, k_{2}, k_{3}\right)+\alpha\left(k_{1}, k_{2}, k_{3}\right) I_{k}\right|^{2}\left(z_{1}^{k_{1}} z_{2}^{k_{2}}+z_{1}^{k_{1}}\right)
$$

and

$$
B_{2}\left(z_{1}, z_{2}, z_{3}\right)=\sum_{\left(k_{1}, k_{2}, k_{3}\right) \in S_{F}}\left|\hat{Q}\left(k_{1}, k_{2}, k_{3}\right)+\alpha\left(k_{1}, k_{2}, k_{3}\right) I_{k}\right|^{2}\left(z_{2}^{k_{2}} z_{3}^{k_{3}}+z_{2}^{k_{2}}\right) .
$$

It is straightforward to see that

$$
\begin{aligned}
\prod_{j=1}^{2} B_{j}\left(z_{1}, z_{2}, z_{3}\right)= & \sum_{\left(k_{1}, k_{2}, k_{3}\right) \in S_{F}}\left|\hat{Q}\left(k_{1}, k_{2}, k_{3}\right)+\alpha\left(k_{1}, k_{2}, k_{3}\right) I_{k}\right|^{4} z_{1}^{k_{1}} z_{2}^{k_{2}} z_{3}^{k_{3}} \\
& +H\left(z_{1}, z_{2}, z_{3}\right) .
\end{aligned}
$$

This implies that there exists a matrix $P^{Q}\left(z_{1}, z_{2}, z_{3}\right) \in M_{k}\left(\mathcal{P}_{3}\right)$ of complex polynomials over $\mathbb{D}^{3}$ such that

$$
\prod_{j=1}^{2} B_{j}\left(Q\left(z_{1}, z_{2}, z_{3}\right)\right)=2 Q\left(z_{1}, z_{2}, z_{3}\right)+P^{Q}\left(z_{1}, z_{2}, z_{3}\right) .
$$

In general, matrices of polynomials over $\mathbb{D}^{n}$ with positive matrices as Fourier coefficients allow the construction of matrices of complex polynomials over $\mathbb{D}^{n}$.

Remark 2.6. Let

$$
F\left(z_{1}, \cdots, z_{n}\right)=\sum_{\left(k_{1}, \cdots, k_{n}\right) \in S_{F}} \hat{F}\left(k_{1}, \cdots, k_{n}\right) z_{1}^{k_{1}} \cdots z_{n}^{k_{n}} \in M_{k}\left(\mathcal{P}_{n}\right)
$$

be a matrix of complex polynomials over $\mathbb{D}^{n}$. Then $\forall\left(k_{1}, \cdots, k_{n}\right) \in S_{F}$ there exists a constant $\alpha\left(k_{1}, \cdots, k_{n}\right) \in \mathbb{N}, \alpha\left(k_{1}, \cdots, k_{n}\right) \neq 0$, such that

$$
\left|4 \hat{F}\left(k_{1}, \cdots, k_{n}\right)+\alpha\left(k_{1}, \cdots, k_{n}\right) I_{k}\right|^{2} \neq 0 \text {. }
$$

Let

$$
\begin{aligned}
& G_{j}\left(F\left(z_{1}, \cdots, z_{n}\right)\right) \\
& =\sum_{\left(k_{1}, \cdots, k_{n}\right) \in S_{F}}\left|4 \hat{F}\left(k_{1}, \cdots, k_{n}\right)+\alpha\left(k_{1}, \cdots, k_{n}\right) I_{k}\right|^{2}\left[z_{j}^{k_{j}} z_{j+1}^{k_{j+1}}+z_{j}^{k_{j}}\right],
\end{aligned}
$$

$j=1, \cdots, n-1$, be matrices of complex polynomials over $\mathbb{D}^{n}$. Then there exists a matrix of complex polynomials $P^{F}\left(z_{1}, \cdots, z_{n}\right)$ over $\mathbb{D}^{n}$ such that

$$
\prod_{j=1}^{n-1} G_{j}\left(F\left(z_{1}, \cdots, z_{n}\right)\right)=2 F\left(z_{1}, \cdots, z_{n}\right)+P^{F}\left(z_{1}, \cdots, z_{n}\right) .
$$

What we need to notice is that the sets

$$
\left\{\left\|F\left(T_{1}, \cdots, T_{n}\right)\right\|_{M_{k}}: F \in M_{k}\left(\mathcal{P}_{n}\right)\right\},\left\{\|F\|_{\infty}: F \in M_{k}\left(\mathcal{P}_{n}\right)\right\}
$$


are not bounded. However, the factorization of matrices of complex polynomials, in terms of the product of matrices of complex polynomials of two variables, did allows us to prove that the set

$$
\left\{\frac{\left\|F\left(T_{1}, \cdots, T_{n}\right)\right\|_{M_{k}}}{\|F\|_{\infty}}: F \in M_{k}\left(\mathcal{P}_{n}\right), F \neq 0\right\}
$$

is bounded by $2^{n}$.

Theorem 2.7. Let $\mathcal{P}_{n}$ be the algebra of complex polynomials over $\mathbb{D}^{n}$ and let $\left\{T_{1}, T_{2}, \cdots, T_{n}\right\} \subset B(H)$ be a set of commuting contractions on the Hilbert space $H$. Then

$$
\left\|F\left(T_{1}, \cdots, T_{n}\right)\right\| \leq 2^{n}\|F\|_{\infty}, \forall F\left(z_{1}, \cdots, z_{n}\right) \in M_{k}\left(\mathcal{P}_{n}\right), k \in \mathbb{N} .
$$

Proof. Let

$$
F\left(z_{1}, \cdots, z_{n}\right)=\sum_{\left(k_{1}, \cdots, k_{n}\right) \in S_{F}} \hat{F}\left(k_{1}, \cdots, k_{n}\right) z_{1}^{k_{1}} \cdots z_{n}^{k_{n}} \in M_{k}\left(\mathcal{P}_{n}\right)
$$

be a matrix of complex polynomials over $\mathbb{D}^{n}$. Then $\forall\left(k_{1}, \cdots, k_{n}\right) \in S_{F}$ there exists a constant $\alpha\left(k_{1}, \cdots, k_{n}\right) \in \mathbb{N}, \alpha\left(k_{1}, \cdots, k_{n}\right) \neq 0$, such that

$$
K=\left|4 \hat{F}\left(k_{1}, \cdots, k_{n}\right)+\alpha\left(k_{1}, \cdots, k_{n}\right) I_{k}\right|^{2} \neq 0 .
$$

Recall that

$$
K=\left[4 \hat{F}\left(k_{1}, \cdots, k_{n}\right)^{*}+\alpha\left(k_{1}, \cdots, k_{n}\right) I_{k}\right]\left[4 \hat{F}\left(k_{1}, \cdots, k_{n}\right)+\alpha\left(k_{1}, \cdots, k_{n}\right) I_{k}\right] .
$$

Denote by

$$
\Delta^{(4)}\left(F\left(z_{1}, \cdots, z_{n}\right)\right)=\sum_{\left(k_{1}, \cdots, k_{n}\right) \in S_{F}}\left|4 \hat{F}\left(k_{1}, \cdots, k_{n}\right)+\alpha\left(k_{1}, \cdots, k_{n}\right) I_{k}\right|^{2} z_{1}^{k_{1}} \cdots z_{n}^{k_{n}}
$$

the matrix of complex polynomials with positive matrices as Fourier coefficients. The matrix of complex polynomials $\Delta^{(4)}\left(F\left(z_{1}, \cdots, z_{n}\right)\right)$ satisfies the von Neumann's inequality. Denote by

$$
\beta_{j}\left(F\left(z_{1}, \cdots, z_{n}\right)\right)=\sum_{\left(k_{1}, \cdots, k_{n}\right) \in S_{F}}\left|4 \hat{F}\left(k_{1}, \cdots, k_{n}\right)+\alpha\left(k_{1}, \cdots, k_{n}\right) I_{k}\right|^{2} z_{j}^{k_{j}} z_{j+1}^{k_{j+1}}
$$

and

$$
\delta_{j}\left(F\left(z_{1}, \cdots, z_{n}\right)\right)=\sum_{\left(k_{1}, \cdots, k_{n}\right) \in S_{F}}\left|4 \hat{F}\left(k_{1}, \cdots, k_{n}\right)+\alpha\left(k_{1}, \cdots, k_{n}\right) I_{k}\right|^{2} z_{j}^{k_{j}},
$$

$j=1, \cdots, n-1$. As we can see $\beta_{j}\left(F\left(z_{1}, \cdots, z_{n}\right)\right), j=1, \cdots, n-1$, are matrices of complex polynomials of two variables with positive matrices as Fourier coefficients and $\delta_{j}\left(F\left(z_{1}, \cdots, z_{n}\right)\right), j=1, \cdots, n-1$, are matrices of complex polynomials of one variable with positive matrices as Fourier coefficients. These polynomials have exactly the same number of Fourier coefficients than $F\left(z_{1}, \cdots, z_{n}\right)$ and they all satisfy the von Neumann inequality. It is straightforward to see that

$$
\left\|\beta_{j}(F)\right\|_{\infty}=\left\|\sum_{\left(k_{1}, \cdots, k_{n}\right) \in S_{F}}\left|4 \hat{F}\left(k_{1}, \cdots, k_{n}\right)+\alpha\left(k_{1}, \cdots, k_{n}\right) I_{k}\right|^{2}\right\|_{M_{k}},
$$




$$
\left\|\delta_{j}(F)\right\|_{\infty}=\left\|\beta_{j}(F)\right\|_{\infty}
$$

and

$$
\left\|\beta_{j}(F)+\delta_{j}(F)\right\|_{\infty}=2\left\|\delta_{j}(F)\right\|_{\infty}=2\left\|\beta_{j}(F)\right\|_{\infty} .
$$

Denote by

$$
G_{j}\left(F\left(z_{1}, \cdots, z_{n}\right)\right)=\beta_{j}\left(F\left(z_{1}, \cdots, z_{n}\right)\right)+\delta_{j}\left(F\left(z_{1}, \cdots, z_{n}\right)\right), j=1, \cdots, n .
$$

That is,

$$
\begin{aligned}
& G_{j}\left(F\left(z_{1}, \cdots, z_{n}\right)\right) \\
& =\sum_{\left(k_{1}, \cdots, k_{n}\right) \in S_{F}}\left|4 \hat{F}\left(k_{1}, \cdots, k_{n}\right)+\alpha\left(k_{1}, \cdots, k_{n}\right) I_{k}\right|^{2}\left(z_{j}^{k_{j}} z_{j+1}^{k_{j+1}}+z_{j}^{k_{j}}\right),
\end{aligned}
$$

$j=1, \cdots, n$. Namelly,

$$
\begin{gathered}
G_{1}\left(F\left(z_{1}, \cdots, z_{n}\right)\right)=\sum_{\left(k_{1}, \cdots, k_{n}\right) \in S_{F}}\left|4 \hat{F}\left(k_{1}, \cdots, k_{n}\right)+\alpha\left(k_{1}, \cdots, k_{n}\right) I_{k}\right|^{2}\left(z_{1}^{k_{1}} z_{2}^{k_{2}}+z_{1}^{k_{1}}\right), \\
G_{2}\left(F\left(z_{1}, \cdots, z_{n}\right)\right)=\sum_{\left(k_{1}, \cdots, k_{n}\right) \in S_{F}}\left|4 \hat{F}\left(k_{1}, \cdots, k_{n}\right)+\alpha\left(k_{1}, \cdots, k_{n}\right) I_{k}\right|^{2}\left(z_{2}^{k_{2}} z_{3}^{k_{3}}+z_{2}^{k_{2}}\right), \\
\vdots \\
G_{r}\left(F\left(z_{1}, \cdots, z_{n}\right)\right)=\sum_{\left(k_{1}, \cdots, k_{n}\right) \in S_{F}}\left|4 \hat{F}\left(k_{1}, \cdots, k_{n}\right)+\alpha\left(k_{1}, \cdots, k_{n}\right) I_{k}\right|^{2}\left(z_{r}^{k_{r}} z_{r+1}^{k_{r+1}}+z_{r}^{k_{r}}\right),
\end{gathered}
$$$$
G_{n-2}\left(F\left(z_{1}, \cdots, z_{n}\right)\right)=\sum_{\left(k_{1}, \cdots, k_{n}\right) \in S_{F}}\left|4 \hat{F}\left(k_{1}, \cdots, k_{n}\right)+\alpha\left(k_{1}, \cdots, k_{n}\right) I_{k}\right|^{2}\left(z_{n-2}^{k_{n-2}} z_{n-1}^{k_{n-1}}+z_{n-2}^{k_{n-2}}\right),
$$$$
G_{n-1}\left(F\left(z_{1}, \cdots, z_{n}\right)\right)=\sum_{\left(k_{1}, \cdots, k_{n}\right) \in S_{F}}\left|4 \hat{F}\left(k_{1}, \cdots, k_{n}\right)+\alpha\left(k_{1}, \cdots, k_{n}\right) I_{k}\right|^{2}\left(z_{n-1}^{k_{n-1}} z_{n}^{k_{n}}+z_{n-1}^{k_{n-1}}\right),
$$

It is not difficult to see that

$$
\begin{aligned}
& \prod_{j=1}^{n-1} G_{j}\left(F\left(z_{1}, \cdots, z_{n}\right)\right) \\
& =\sum_{\left(k_{1}, \cdots, k_{n}\right) \in S_{F}}\left|\hat{F}\left(k_{1}, \cdots, k_{n}\right)+\alpha\left(k_{1}, \cdots, k_{n}\right) I_{k}\right|^{2(n-1)} z_{1}^{k_{1}} \cdots z_{n}^{k_{n}}+Q^{F}\left(z_{1}, \cdots, z_{n}\right) .
\end{aligned}
$$

This implies that there exists a matrix $P^{F}\left(z_{1}, \cdots, z_{n}\right) \in M_{k}\left(\mathcal{P}_{n}\right)$ of complex polynomials over $\mathbb{D}^{n}$ such that

$$
\prod_{j=1}^{n-1} G_{j}\left(F\left(z_{1}, \cdots, z_{n}\right)\right)=2 F\left(z_{1}, \cdots, z_{n}\right)+P^{F}\left(z_{1}, \cdots, z_{n}\right) .
$$

In other words,

$$
2 F\left(z_{1}, \cdots, z_{n}\right)+P^{F}\left(z_{1}, \cdots, z_{n}\right)=\prod_{j=1}^{n-1}\left[\beta_{j}\left(F\left(z_{1}, \cdots, z_{n}\right)\right)+\delta_{j}\left(F\left(z_{1}, \cdots, z_{n}\right)\right)\right] .
$$

It is straightforward to see that

$$
\left\|\prod_{j=1}^{n-1} G_{j}(F)\right\|_{\infty}=\left\|2 F+P^{F}\right\|_{\infty}=\prod_{j=1}^{n-1}\left\|\beta_{j}(F)+\delta_{j}(F)\right\|_{\infty} .
$$

In general, the matrix $P^{F}\left(z_{1}, \cdots, z_{n}\right)$ is not unique. The structure of the ma- 
trix $P^{F}\left(z_{1}, \cdots, z_{n}\right)$ depends on the structure of $\Delta^{(4)}\left(F\left(z_{1}, \cdots, z_{n}\right)\right)$. Let $V=\left(T_{1}, T_{2}, \cdots, T_{n}\right)$ be an $n$-tuple of commuting contractions on a Hilbert space $\mathrm{H}$. The von Neumann inequality for complex polynomials of two (or one) variable(s) allows us to say that

$$
\frac{\left\|\beta_{j}\left(F\left(T_{1}, \cdots, T_{n}\right)\right)\right\|}{\left\|\beta_{j}(F)\right\|_{\infty}} \leq 1, \frac{\left\|\delta_{j}\left(F\left(T_{1}, \cdots, T_{n}\right)\right)\right\|}{\left\|\delta_{j}(F)\right\|_{\infty}} \leq 1 .
$$

This implies that

$$
\frac{\left\|\beta_{j}\left(F\left(T_{1}, \cdots, T_{n}\right)\right)\right\|}{\left\|\beta_{j}(F)\right\|_{\infty}}+\frac{\left\|\delta_{j}\left(F\left(T_{1}, \cdots, T_{n}\right)\right)\right\|}{\left\|\delta_{j}(F)\right\|_{\infty}} \leq 2 .
$$

Suppose that

$$
G^{F}\left(z_{1}, \cdots, z_{n}\right)=\prod_{j=1}^{n-1} G_{j}\left(F\left(z_{1}, \cdots, z_{n}\right)\right)=2 F\left(z_{1}, \cdots, z_{n}\right)+P^{F}\left(z_{1}, \cdots, z_{n}\right) .
$$

It follows that

$$
G^{F}\left(T_{1}, \cdots, T_{n}\right)=\prod_{j=1}^{n-1}\left[\beta_{j}\left(F\left(T_{1}, \cdots, T_{n}\right)\right)+\delta_{j}\left(F\left(T_{1}, \cdots, T_{n}\right)\right)\right] .
$$

This implies that,

$$
\left\|G^{F}\left(T_{1}, \cdots, T_{n}\right)\right\|=\left\|\prod_{j=1}^{n-1}\left[\beta_{j}\left(F\left(T_{1}, \cdots, T_{n}\right)\right)+\delta_{j}\left(F\left(T_{1}, \cdots, T_{n}\right)\right)\right]\right\| .
$$

Thus,

$$
\left\|G^{F}\left(T_{1}, \cdots, T_{n}\right)\right\| \leq \prod_{j=1}^{n-1}\left\|\left[\beta_{j}\left(F\left(T_{1}, \cdots, T_{n}\right)\right)+\delta_{j}\left(F\left(T_{1}, \cdots, T_{n}\right)\right)\right]\right\| .
$$

Also, we have

$$
\left\|G^{F}\left(T_{1}, \cdots, T_{n}\right)\right\| \leq \prod_{j=1}^{n-1}\left[\left\|\beta_{j}\left(F\left(T_{1}, \cdots, T_{n}\right)\right)\right\|+\left\|\delta_{j}\left(F\left(T_{1}, \cdots, T_{n}\right)\right)\right\|\right] .
$$

It is easy to say that

$$
\frac{\left\|G^{F}\left(T_{1}, \cdots, T_{n}\right)\right\|}{\left\|G^{F}\right\|_{\infty}} \leq \prod_{j=1}^{n-1}\left[\frac{\left\|\beta_{j}\left(F\left(T_{1}, \cdots, T_{n}\right)\right)\right\|+\left\|\delta_{j}\left(F\left(T_{1}, \cdots, T_{n}\right)\right)\right\|}{\left\|\beta_{j}(F)+\delta_{j}(F)\right\|_{\infty}}\right] .
$$

Therefore,

$$
\frac{\left\|G^{F}\left(T_{1}, \cdots, T_{n}\right)\right\|}{\left\|G^{F}\right\|_{\infty}} \leq \prod_{j=1}^{n-1}\left[\frac{\left\|\beta_{j}\left(F\left(T_{1}, \cdots, T_{n}\right)\right)\right\|}{\left\|\beta_{j}(F)+\delta_{j}(F)\right\|_{\infty}}+\frac{\left\|\delta_{j}\left(F\left(T_{1}, \cdots, T_{n}\right)\right)\right\|}{\left\|\beta_{j}(F)+\delta_{j}(F)\right\|_{\infty}}\right] .
$$

We can claim that

$$
\frac{\left\|G^{F}\left(T_{1}, \cdots, T_{n}\right)\right\|}{\left\|G^{F}\right\|_{\infty}} \leq \prod_{j=1}^{n-1}\left[\frac{\left\|\beta_{j}\left(F\left(T_{1}, \cdots, T_{n}\right)\right)\right\|}{\left\|\beta_{j}(F)\right\|_{\infty}}+\frac{\left\|\delta_{j}\left(F\left(T_{1}, \cdots, T_{n}\right)\right)\right\|}{\left\|\delta_{j}(F)\right\|_{\infty}}\right],
$$

since, in general,

$$
\left\|\delta_{j}(F)\right\|_{\infty} \leq\left\|\beta_{j}(F)+\delta_{j}(F)\right\|_{\infty},\left\|\beta_{j}(F)\right\|_{\infty} \leq\left\|\beta_{j}(F)+\delta_{j}(F)\right\|_{\infty} .
$$

It is clear that 


$$
\frac{\left\|G^{F}\left(T_{1}, \cdots, T_{n}\right)\right\|}{\left\|G^{F}\right\|_{\infty}} \leq \prod_{j=1}^{n-1}[1+1]=2^{n-1}
$$

This implies that

$$
\sup \left\{\frac{\left\|2 F\left(T_{1}, \cdots, T_{n}\right)+P^{F}\left(T_{1}, \cdots, T_{n}\right)\right\|}{\left\|2 F+P^{F}\right\|_{\infty}}: F \in M_{k}\left(\mathcal{P}_{n}\right), F \neq 0\right\} \leq 2^{n-1},
$$

since $G^{F}\left(z_{1}, \cdots, z_{n}\right)=2 F\left(z_{1}, \cdots, z_{n}\right)+P^{F}\left(z_{1}, \cdots, z_{n}\right)$. Due to the fact that

$$
\left\|2 F\left(T_{1}, \cdots, T_{n}\right)+P^{F}\left(T_{1}, \cdots, T_{n}\right)\right\| \leq\left\|2 F\left(T_{1}, \cdots, T_{n}\right)\right\|+\left\|P^{F}\left(T_{1}, \cdots, T_{n}\right)\right\|
$$

and

$$
\left\|2 F+P^{F}\right\|_{\infty} \leq\|2 F\|_{\infty}+\left\|P^{F}\right\|_{\infty},
$$

we can say that for every $F \in M_{k}\left(\mathcal{P}_{n}\right), F \neq 0$, there exist two positive real numbers $\alpha_{F}^{V}$ and $\lambda_{F}$ such that

$$
\left\|2 F\left(T_{1}, \cdots, T_{n}\right)+P^{F}\left(T_{1}, \cdots, T_{n}\right)\right\|+\alpha_{F}^{V}=\left\|2 F\left(T_{1}, \cdots, T_{n}\right)\right\|+\left\|P^{F}\left(T_{1}, \cdots, T_{n}\right)\right\|
$$

and

$$
\left\|2 F+P^{F}\right\|_{\infty}+\lambda_{F}=\|2 F\|_{\infty}+\left\|P^{F}\right\|_{\infty} .
$$

Thus,

$$
\left\|2 F\left(T_{1}, \cdots, T_{n}\right)+P^{F}\left(T_{1}, \cdots, T_{n}\right)\right\|=\left\|2 F\left(T_{1}, \cdots, T_{n}\right)\right\|+\left\|P^{F}\left(T_{1}, \cdots, T_{n}\right)\right\|-\alpha_{F}^{V}
$$

and

$$
\left\|2 F+P^{F}\right\|_{\infty}=\|2 F\|_{\infty}+\left\|P^{F}\right\|_{\infty}-\lambda_{F} .
$$

Therefore,

$$
\frac{\left\|2 F\left(T_{1}, \cdots, T_{n}\right)+P^{F}\left(T_{1}, \cdots, T_{n}\right)\right\|}{\left\|2 F+P^{F}\right\|_{\infty}}=\frac{\left\|2 F\left(T_{1}, \cdots, T_{n}\right)\right\|+\left\|P^{F}\left(T_{1}, \cdots, T_{n}\right)\right\|-\alpha_{F}^{V}}{\|2 F\|_{\infty}+\left\|P^{F}\right\|_{\infty}-\lambda_{F}} .
$$

It is not difficult to see that

$$
\frac{\left\|2 F\left(T_{1}, \cdots, T_{n}\right)\right\|+\left\|P^{F}\left(T_{1}, \cdots, T_{n}\right)\right\|-\alpha_{F}^{V}}{\|2 F\|_{\infty}+\left\|P^{F}\right\|_{\infty}} \leq \frac{\left\|2 F\left(T_{1}, \cdots, T_{n}\right)+P^{F}\left(T_{1}, \cdots, T_{n}\right)\right\|}{\left\|2 F+P^{F}\right\|_{\infty}} .
$$

Now, we can claim that

$$
\sup \left\{\frac{\left\|2 F\left(T_{1}, \cdots, T_{n}\right)\right\|+\left\|P^{F}\left(T_{1}, \cdots, T_{n}\right)\right\|-\alpha_{F}^{V}}{\|2 F\|_{\infty}+\left\|P^{F}\right\|_{\infty}}: F \in M_{k}\left(\mathcal{P}_{n}\right), F \neq 0\right\} \leq 2^{n-1} .
$$

The Archimedean property of the total order on $\mathbb{R}$ allows us to say that there exists a positive real number $d\left(P^{F}\right)$ such that

$$
\left\|P^{F}\left(T_{1}, \cdots, T_{n}\right)\right\|+\theta_{P^{F}}^{V}=d\left(P^{F}\right)\left\|P^{F}\right\|_{\infty}, \theta_{P^{F}}^{V} \in \mathbb{R}^{+},
$$

where $d(F) \neq 1$ is the smallest positive integer such that 


$$
\left\|P^{F}\left(T_{1}, \cdots, T_{n}\right)\right\| \leq \sum_{\left(k_{1}, \cdots, k_{n}\right) \in S_{P^{F}}}\left\|\hat{P}^{F}\left(k_{1}, \cdots, k_{n}\right)\right\|_{M_{k}} \leq d(F)\left\|P^{F}\right\|_{\infty} .
$$

This allows us to say that

$$
\sup \left\{\frac{\left\|2 F\left(T_{1}, \cdots, T_{n}\right)\right\|+d\left(P^{F}\right)\left\|P^{F}\right\|_{\infty}-\theta_{P^{F}}^{V}-\alpha_{F}^{V}}{\|2 F\|_{\infty}+\left\|P^{F}\right\|_{\infty}}: F \in M_{k}\left(\mathcal{P}_{n}\right), F \neq 0\right\} \leq 2^{n-1} .
$$

It follows that

$$
\sup \left\{\frac{\left\|2 F\left(T_{1}, \cdots, T_{n}\right)\right\|+d\left(P^{F}\right)\left\|P^{F}\right\|_{\infty}-\theta_{P^{F}}^{V}-\alpha_{F}^{V}}{\|2 F\|_{\infty}+d\left(P^{F}\right)\left\|P^{F}\right\|_{\infty}}: F \in M_{k}\left(\mathcal{P}_{n}\right), F \neq 0\right\} \leq 2^{n-1},
$$

since $\left(\|2 F\|_{\infty}+\left\|P^{F}\right\|_{\infty} \leq\|2 F\|_{\infty}+d\left(P^{F}\right)\left\|P^{F}\right\|_{\infty}\right)$. In other words,

$$
\sup \left\{\frac{2\left\|F\left(T_{1}, \cdots, T_{n}\right)\right\|+d\left(P^{F}\right)\left\|P^{F}\right\|_{\infty}-\omega_{F}^{V}}{2\|F\|_{\infty}+d\left(P^{F}\right)\left\|P^{F}\right\|_{\infty}}: F \in M_{k}\left(\mathcal{P}_{n}\right), F \neq 0\right\} \leq 2^{n-1},
$$

with $\omega_{F}^{V}=\theta_{P^{F}}^{V}+\alpha_{F}^{V} \geq 0$. Let us notice that

$$
0 \leq \frac{2\left\|F\left(T_{1}, \cdots, T_{n}\right)\right\|+d\left(P^{F}\right)\left\|P^{F}\right\|_{\infty}-\omega_{F}^{V}}{2\|F\|_{\infty}+d\left(P^{F}\right)\left\|P^{F}\right\|_{\infty}}, F \in M_{k}\left(\mathcal{P}_{n}\right), F \neq 0 .
$$

This implies that

$$
0 \leq \omega_{F}^{V} \leq 2\left\|F\left(T_{1}, \cdots, T_{n}\right)\right\|+d\left(P^{F}\right)\left\|P^{F}\right\|_{\infty}, F \in M_{k}\left(\mathcal{P}_{n}\right), F \neq 0 .
$$

This means that

$$
0 \leq \frac{\omega_{F}^{V}}{2\left\|F\left(T_{1}, \cdots, T_{n}\right)\right\|+d\left(P^{F}\right)\left\|P^{F}\right\|_{\infty}} \leq 1 \leq 2^{n-1}, F \in M_{k}\left(\mathcal{P}_{n}\right), F \neq 0 .
$$

Also, we can notice that

$$
0 \leq \frac{\omega_{F}^{V}}{2\|F\|_{\infty}+d\left(P^{F}\right)\left\|P^{F}\right\|_{\infty}} \leq 2^{n-1}, F \in M_{k}\left(\mathcal{P}_{n}\right), F \neq 0 .
$$

It is straightforward to observe that if we add the equations (2.1) and (2.3), one has

$$
0 \leq \frac{2\left\|F\left(T_{1}, \cdots, T_{n}\right)\right\|+d\left(P^{F}\right)\left\|P^{F}\right\|_{\infty}}{2\|F\|_{\infty}+d\left(P^{F}\right)\left\|P^{F}\right\|_{\infty}} \leq 2^{n}, F \in M_{k}\left(\mathcal{P}_{n}\right), F \neq 0 .
$$

We can claim now that

$$
\mathcal{B}_{n}=\sup \left\{\frac{\left\|2 F\left(T_{1}, \cdots, T_{n}\right)\right\|+d\left(P^{F}\right)\left\|P^{F}\right\|_{\infty}}{\|2 F\|_{\infty}+d\left(P^{F}\right)\left\|P^{F}\right\|_{\infty}}: F \in M_{k}\left(\mathcal{P}_{n}\right), F \neq 0\right\} \leq 2^{n} .
$$

It follows that

$$
0 \leq \frac{\left\|2 F\left(T_{1}, \cdots, T_{n}\right)\right\|+d\left(P^{F}\right)\left\|P^{F}\right\|_{\infty}}{\|2 F\|_{\infty}+d\left(P^{F}\right)\left\|P^{F}\right\|_{\infty}} \leq 2^{n}, \forall F \in M_{k}\left(\mathcal{P}_{n}\right) .
$$


We can say that the set

$$
\left\{\frac{\left\|2 F\left(T_{1}, \cdots, T_{n}\right)\right\|+d\left(P^{F}\right)\left\|P^{F}\right\|_{\infty}}{\|2 F\|_{\infty}+d\left(P^{F}\right)\left\|P^{F}\right\|_{\infty}}: F \in M_{k}\left(\mathcal{P}_{n}\right), F \neq 0\right\}
$$

is bounded. The set

$$
\left\{\frac{\left\|2 F\left(T_{1}, \cdots, T_{n}\right)\right\|}{\|2 F\|_{\infty}}: F \in M_{k}\left(\mathcal{P}_{n}\right), F \neq 0\right\}
$$

is also bounded. Indeed, assume that there exists $F_{0} \in M_{k}\left(\mathcal{P}_{n}\right), F_{0} \neq 0$ such that

$$
\frac{\left\|2 F_{0}\left(T_{1}, \cdots, T_{n}\right)\right\|}{\left\|2 F_{0}\right\|_{\infty}}=+\infty
$$

This implies that

$$
\frac{\left\|2 F_{0}\left(T_{1}, \cdots, T_{n}\right)\right\|+d\left(P^{F_{0}}\right)\left\|P^{F_{0}}\right\|_{\infty}}{\left\|2 F_{0}\right\|_{\infty}+d\left(P^{F_{0}}\right)\left\|P^{F_{0}}\right\|_{\infty}}=+\infty .
$$

We have a contradiction because

$$
0 \leq \frac{\left\|2 F_{0}\left(T_{1}, \cdots, T_{n}\right)\right\|+d\left(P^{F_{0}}\right)\left\|P^{F_{0}}\right\|_{\infty}}{\left\|2 F_{0}\right\|_{\infty}+d\left(P^{F_{0}}\right)\left\|P^{F_{0}}\right\|_{\infty}} \leq 2^{n} .
$$

Therefore, there exists a positive constant $K_{n}$ such that

$$
0 \leq \frac{\left\|F\left(T_{1}, \cdots, T_{n}\right)\right\|}{\|F\|_{\infty}} \leq K_{n}, \forall F \in M_{k}\left(\mathcal{P}_{n}\right), F \neq 0 .
$$

Suppose that

$$
\mathcal{A}_{n}=\sup \left\{\frac{F\left(T_{1}, \cdots, T_{n}\right)}{\|F\|_{\infty}}: F \in M_{k}\left(\mathcal{P}_{n}\right), F \neq 0\right\} .
$$

It is clear that $\mathcal{A}_{n} \leq K_{n}$. Let us estimate $\mathcal{A}_{n}$. Assume that $\left\|F\left(T_{1}, \cdots, T_{n}\right)\right\| \leq\|F\|_{\infty}$. One has

$$
\frac{\left\|2 F\left(T_{1}, \cdots, T_{n}\right)\right\|+d\left(P^{F}\right)\left\|P^{F}\right\|_{\infty}}{\|2 F\|_{\infty}+d\left(P^{F}\right)\left\|P^{F}\right\|_{\infty}} \leq \frac{\left\|2 F\left(T_{1}, \cdots, T_{n}\right)\right\|+d\left(P^{F}\right)\left\|P^{F}\right\|_{\infty}+\|Q\|_{\infty}}{\|2 F\|_{\infty}+d\left(P^{F}\right)\left\|P^{F}\right\|_{\infty}+\|Q\|_{\infty}} \leq 1,
$$

$\forall Q \in M_{k}\left(\mathcal{P}_{n}\right)$. Now, assume that $\|F\|_{\infty} \leq\left\|F\left(T_{1}, \cdots, T_{n}\right)\right\|$. We have

$$
1 \leq \frac{\left\|2 F\left(T_{1}, \cdots, T_{n}\right)\right\|+d\left(P^{F}\right)\left\|P^{F}\right\|_{\infty}+\|Q\|_{\infty}}{\|2 F\|_{\infty}+d\left(P^{F}\right)\left\|P^{F}\right\|_{\infty}+\|Q\|_{\infty}} \leq \frac{\left\|2 F\left(T_{1}, \cdots, T_{n}\right)\right\|+d\left(P^{F}\right)\left\|P^{F}\right\|_{\infty}}{\|2 F\|_{\infty}+d\left(P^{F}\right)\left\|P^{F}\right\|_{\infty}},
$$

$\forall Q \in M_{k}\left(\mathcal{P}_{n}\right)$. Therefore, for every $Q \in M_{k}\left(\mathcal{P}_{n}\right)$, we have

$$
0 \leq \frac{\left\|2 F\left(T_{1}, \cdots, T_{n}\right)\right\|+d\left(P^{F}\right)\left\|P^{F}\right\|_{\infty}+\|Q\|_{\infty}}{\|2 F\|_{\infty}+d\left(P^{F}\right)\left\|P^{F}\right\|_{\infty}+\|Q\|_{\infty}} \leq 2^{n}, \forall F \in M_{k}\left(\mathcal{P}_{n}\right), F \neq 0 .
$$

The equation (2.6) allows us to say that for every $Q \in M_{k}\left(\mathcal{P}_{n}\right)$, we have 


$$
0 \leq \frac{\left\|2 F\left(T_{1}, \cdots, T_{n}\right)\right\|+\left\|Q+d\left(P^{F}\right)\right\| P^{F}\left\|_{\infty} I_{k}\right\|_{\infty}}{\|2 F\|_{\infty}+\left\|Q+d\left(P^{F}\right)\right\| P^{F}\left\|_{\infty} I_{k}\right\|_{\infty}} \leq 2^{n}, \forall F \in M_{k}\left(\mathcal{P}_{n}\right), F \neq 0 .
$$

In particular, if we choose the matrix $Q=-d\left(P^{F}\right)\left\|P^{F}\right\|_{\infty} I_{k}$, one has

$$
0 \leq \frac{\left\|2 F\left(T_{1}, \cdots, T_{n}\right)\right\|}{\|2 F\|_{\infty}} \leq 2^{n}, \forall F \in M_{k}\left(\mathcal{P}_{n}\right), F \neq 0 .
$$

In other words,

$$
\mathcal{A}_{n}=\sup \left\{\frac{\left\|F\left(T_{1}, \cdots, T_{n}\right)\right\|}{\|F\|_{\infty}}: F \in M_{k}\left(\mathcal{P}_{n}\right), F \neq 0\right\} \leq 2^{n} .
$$

Finally,

$$
\left\|F\left(T_{1}, \cdots, T_{n}\right)\right\| \leq 2^{n}\|F\|_{\infty}, \forall F \in M_{k}\left(\mathcal{P}_{n}\right) .
$$

Let us observe that if

$$
0 \leq \frac{\left\|F\left(T_{1}, \cdots, T_{n}\right)\right\|}{\|F\|_{\infty}} \leq 2^{n}, \forall F \in M_{k}\left(\mathcal{P}_{n}\right), F \neq 0,
$$

then for every $Q \in M_{k}\left(\mathcal{P}_{n}\right)$, one has

$$
0 \leq \frac{\left\|F\left(T_{1}, \cdots, T_{n}\right)\right\|+\|Q\|_{\infty}}{\|F\|_{\infty}+\|Q\|_{\infty}} \leq 2^{n}, \forall F \in M_{k}\left(\mathcal{P}_{n}\right), F \neq 0 .
$$

In particular, for $Q=d\left(P^{F}\right)\left\|P^{F}\right\|_{\infty} I_{k}$, one has

$$
0 \leq \frac{\left\|F\left(T_{1}, \cdots, T_{n}\right)\right\|+d\left(P^{F}\right)\left\|P^{F}\right\|_{\infty}}{\|F\|_{\infty}+d\left(P^{F}\right)\left\|P^{F}\right\|_{\infty}} \leq 2^{n}, \forall F \in M_{k}\left(\mathcal{P}_{n}\right), F \neq 0 .
$$

In fact, $\mathcal{A}_{n}=\mathcal{B}_{n}$. Theorem 2.7, Theorem 2.1 and Theorem 2.2 allow us to prove our main result. Every homomorphism of the algebra $\mathcal{P}_{n}$ is completely bounded.

Theorem 2.8. Let $\mathcal{P}_{n}$ be the algebra of complex polynomials over $\mathbb{D}^{n}$, let $\mathbb{V}=\left\{T_{1}, T_{2}, \cdots, T_{n}\right\} \subset B(H)$ be a set of commuting contractions on the Hilbert space $H$ and let $\phi^{\mathbb{V}}: \mathcal{P}_{n} \rightarrow B(H)$ be the map given by

$$
\phi^{\mathbb{V}}(f)=f\left(T_{1}, \cdots, T_{n}\right) .
$$

Then there exists a homomorphism $\Gamma^{\mathbb{V}}: C\left(\mathbb{T}^{n}\right) \rightarrow B(H)$ similar to a *-homomorphism such that

$$
\Gamma^{\mathbb{V}}(f)=\phi^{\mathbb{V}}(f), \forall f \in \mathcal{P}_{n} .
$$

Proof. Let $\mathcal{P}_{n}$ be the algebra of complex polynomials over $\mathbb{D}^{n}$ and let $\mathbb{V}=\left\{T_{1}, T_{2}, \cdots, T_{n}\right\} \subset B(H)$ be a set of commuting contractions on the Hilbert space $\mathrm{H}$. We just need to show that the map $\phi^{\mathbb{V}}: \mathcal{P}_{n} \rightarrow B(H)$ given by

$$
\phi^{\mathbb{V}}(f)=f\left(T_{1}, \cdots, T_{n}\right) .
$$

is a completely bounded homomorphism. It is well known that this map is a 
homomorphism. Let us show that the map

$$
\phi_{k}^{\mathbb{V}}: M_{k}\left(\mathcal{P}_{n}\right) \rightarrow M_{k}(B(H))
$$

given by

$$
\phi_{k}^{\mathbb{V}}(F)=F\left(T_{1}, \cdots, T_{n}\right)
$$

is bounded for all $k \in \mathbb{N}$ with

$$
F\left(z_{1}, \cdots, z_{n}\right)=\sum_{\left(k_{1}, \cdots, k_{n}\right) \in S_{F}} \hat{F}\left(k_{1}, \cdots, k_{n}\right) z_{1}^{k_{1}} \cdots z_{n}^{k_{n}} \in M_{k}\left(\mathcal{P}_{n}\right) .
$$

Theorem 2.7 allows us to claim that

$$
\sup \left\{\frac{\left\|F\left(T_{1}, \cdots, T_{n}\right)\right\|}{\|F\|_{\infty}}: F \in M_{k}\left(\mathcal{P}_{n}\right), F \neq 0\right\} \leq 2^{n} .
$$

It is well known that

$$
\left\|\phi_{k}^{\mathbb{V}}\right\|=\sup \left\{\frac{\left\|\phi_{k}^{\mathbb{V}}(F)\right\|}{\|F\|_{\infty}}: F \in M_{k}\left(\mathcal{P}_{n}\right), F \neq 0\right\} .
$$

In other words,

$$
\left\|\phi_{k}^{\mathbb{V}}\right\|=\sup \left\{\frac{\left\|F\left(T_{1}, \cdots, T_{n}\right)\right\|}{\|F\|_{\infty}}: F \in M_{k}\left(\mathcal{P}_{n}\right), F \neq 0\right\} .
$$

It follows that

$$
\left\|\phi_{k}^{\mathbb{V}}\right\| \leq 2^{n}, \forall k \in \mathbb{N} .
$$

Therefore,

$$
\sup \left\{\left\|\phi_{k}^{\mathbb{V}}\right\|: k \in \mathbb{N}\right\} \leq 2^{n} .
$$

Finally, the homomorphism $\phi^{\mathbb{V}}$ is completely bounded. Theorem 2.1 enables us to say that there exists a completely bounded homomorphism $\Gamma^{\mathbb{V}}: C\left(\mathbb{T}^{n}\right) \rightarrow B(H)$ such that

$$
\Gamma^{\mathbb{V}}(f)=\phi^{\mathbb{V}}(f), \forall f \in \mathcal{P}_{n},\left\|\Gamma^{\mathbb{V}}\right\|_{c b}=\left\|\phi^{\mathbb{V}}\right\|_{c b} .
$$

Theorem 2.2 allows us to claim that the homomorphism $\Gamma^{\mathbb{V}}$ is similar to a *-homomorphism. In other words, there exists an invertible operator

$$
S: H \rightarrow H
$$

such that $\rho=S^{-1} \Gamma^{\mathbb{V}} S$ is a ${ }^{*}$-homomorphism. In particular,

$$
\phi^{\mathbb{V}}(f)=\Gamma^{\mathbb{V}}(f)=S \rho(f) S^{-1}, \forall f \in \mathcal{P}_{n} .
$$

In general, the properties of every homomorphism of the algebra $\mathcal{P}_{n}$ depend on the structure of the associated tuple $\mathbb{V}=\left\{T_{1}, \cdots, T_{n}\right\}$ which generates that homomorphism. For instance, if the tuple $\mathbb{V}=\left\{T_{1}, \cdots, T_{n}\right\}$ is a doubly commuting set of commuting contractions (or circulant contractions, complex triangular Toeplitz contractions) the corresponding homomorphism $\phi^{\mathbb{V}}: \mathcal{P}_{n} \rightarrow B(H)$ given by $\phi^{\mathbb{V}}(f)=f\left(T_{1}, \cdots, T_{n}\right)$ is completely contractive [16], since von Neumann's inequality hold for this type of tuples. 
Theorem 2.8 and Theorem 2.3 allow us to show that the contractive homomorphism introduced by Parrott in 1970, which is not completely contractive, is similar to a completely contractive homomorphism (completely bounded).

Proposition 2.9. Let $U$ and $V$ be contractions in $B(H)$ such that $U$ is unitary and $U$ and $V$ don't commute. We define commuting contraction on $B(H \oplus H)$ by setting

$$
T_{1}=\left[\begin{array}{ll}
0 & 0 \\
I & 0
\end{array}\right], T_{2}=\left[\begin{array}{cc}
0 & 0 \\
U & 0
\end{array}\right], T_{3}=\left[\begin{array}{cc}
0 & 0 \\
V & 0
\end{array}\right] .
$$

Let $\mathcal{P}_{3}$ be the algebra of complex polynomials over $\mathbb{D}^{3}$, let $\mathbb{V}=\left\{T_{1}, T_{2}, T_{3}\right\}$ be a set of commuting contractions on the Hilbert space $\mathrm{H}$. Then the homomorphism

$$
\phi^{\mathbb{V}}: \mathcal{P}_{3} \rightarrow B(H \oplus H)
$$

given by $\phi^{\mathbb{V}}\left(z_{i}\right)=T_{i}, i=1,2,3$ is a completely bounded homomorphism. Moreover, $\|\rho\|_{c b} \leq 8$.

Proof. Theorem 2.8 and Theorem 2.3 allow us to claim that the homomorphism $\phi^{\mathbb{V}}$ is a completely bounded homomorphism, similar to a completely contractive homomorphism and

$$
\left\|\phi^{\mathbb{V}}\right\|_{c b} \leq 8
$$

We can now introduce the existence of completely positive maps over the $C$-algebras $C\left(\mathbb{T}^{n}\right)$ and $M_{2}\left(C\left(\mathbb{T}^{n}\right)\right)$. Every homomorphism of the algebra $\mathcal{P}_{n}$ generates completely positive maps over the algebras $C\left(\mathbb{T}^{n}\right)$ and $M_{2}\left(C\left(\mathbb{T}^{n}\right)\right)$.

Theorem 2.10. Let $\mathbb{V}=\left\{T_{1}, T_{2}, \cdots, T_{n}\right\} \subset B(H)$ be a set of commuting contractions on the Hilbert space $H$ and let $\phi^{\mathbb{V}}: \mathcal{P}_{n} \rightarrow B(H)$ be the homomorphim given by $\phi^{\mathbb{V}}(f)=f\left(T_{1}, \cdots, T_{n}\right), \forall f \in \mathcal{P}_{n}$. Then there exist completely positive maps

$$
\theta_{i}: C\left(\mathbb{T}^{n}\right) \rightarrow B(H),
$$

with $\left\|\theta_{i}\right\|_{c b}=\|\phi\|_{c b}, i=1,2$ such that the map $\psi: M_{2}\left(C\left(\mathbb{T}^{n}\right)\right) \rightarrow B(H \oplus H)$ given by

$$
\psi\left[\left[\begin{array}{ll}
a & b \\
c & d
\end{array}\right]\right]=\left[\begin{array}{cc}
\theta_{1}(a) & \phi^{\mathbb{V}}(b) \\
\left(\phi^{\mathbb{V}}\right)^{*}(c) & \theta_{2}(d)
\end{array}\right], a, b, c, d \in \mathcal{P}_{n},
$$

is completely positive.

Proof. Theorem 2.8 and Theorem 2.2 allow us to claim that homomorphism $\phi^{\mathbb{V}}$ is completely bounded. Theorem 2.1 enables us to say that there exists a completely bounded homomorphism $\Gamma^{\mathbb{V}}: C\left(\mathbb{T}^{n}\right) \rightarrow B(H)$ such that

$$
\Gamma^{\mathbb{V}}(f)=\phi^{\mathbb{V}}(f), \forall f \in \mathcal{P}_{n},\left\|\Gamma^{\mathbb{V}}\right\|_{c b}=\left\|\phi^{\mathbb{V}}\right\|_{c b} \text {. }
$$

Theorem 2.4 yields the desired result. 


\section{Conflicts of Interest}

The author declares no conflicts of interest regarding the publication of this paper.

\section{References}

[1] Dixmier, J. (1950) Les moyennes invariante dans les semi-groupes et leurs applications. Acta Scientiarum Mathematicarum, 12, 213-227.

[2] Ehrenpreis, L. and Mautner, F. (1955) Uniformly Bounded Representations of Groups. Proceedings of the National Academy of Sciences, 41, 231-233. https://doi.org/10.1073/pnas.41.4.231

[3] Kadison, R.V. (1955) On the Orthogonalization of Operator Representations. American Journal of Mathematics, 77, 600-620. https://doi.org/10.2307/2372645

[4] Kunze, R.A. and Stein, E.M. (1960) Uniformly Bounded Representations and Harmonic Analysis of $2 \times 2$ Real Unimodular Group. American Journal of Mathematics, 82, 1-62. https://doi.org/10.2307/2372876

[5] Paulsen, V.I. (1984) Completely Bounded Homomorphisms of Operator Algebras. Proceedings of the American Mathematical Society, 92, 225-228. https://doi.org/10.1090/S0002-9939-1984-0754708-X

[6] Christensen, E. (1981) On Non Self-Adjoint Representations of $C$-Algebras. American Journal of Mathematics, 103, 817-833. https://doi.org/10.2307/2374248

[7] Haagerup, U. (1983) Solution of the Similarity Problem for Cyclic Representations of $C$-Algebras. Annals of Mathematics, 118, 215-240. https://doi.org/10.2307/2007028

[8] Paulsen, V.I. (1986) Completely Bounded Maps and Dilations (Pitman Research Notes in Mathematics Series 146), Pitman Longman, Wiley City.

[9] Parrott, S.K. (1970) Unitary Dilations for Commuting Contractions. Pacific Journal of Mathematics, 34, 481-490. https://doi.org/10.2140/pjm.1970.34.481

[10] Christensen, E. (1986) Similarities of $I I_{1}$ Factors with Property $\Gamma$. Journal of Operator Theory, 15, 281-288.

[11] Kirchberg, E. (1996) The Derivation Problem and the Similarity Problem Are Equivalent. Journal of Operator Theory, 36, 59-62.

[12] Pisier, G. (1999) The Similarity Degree of an Operator Algebra. St. Petersburg Mathematical Journal, 10, 103-146.

[13] Pisier, G. (2001) Remarks on the Similarity Degree of an Operator Algebra. International Journal of Mathematics, 12, 403-414. https://doi.org/10.1142/S0129167X01000824

[14] Pisier, G. (2006) A Similarity Degree Characterization of Nuclear C-Algebras. Publications of the Research Institute for Mathematical Sciences, 42, 691-704. https://doi.org/10.2977/prims/1166642155

[15] Christensen, E., Sinclair, A., Smith, R.R. and White, S. (2010) Perturbations of C-Algebraic Invariants. Geometric and Functional Analysis, 20, 368-397. https://doi.org/10.1007/s00039-010-0070-y

[16] Mouanda, J.M. (2020) On Von Neumann's Inequality for Tuples of Complex Triangular Toeplitz Contractions. Rocky Mountain Journal of Mathematics, 50, 213-224. https://doi.org/10.1216/rmj.2020.50.213 